\title{
Spin Density Calculations on the Tetraphenylverdazyl Radical and Two Nitroxide Radicals: First and Second Order Spin Polarization
}

\author{
Lars Öhrström, ${ }^{a}, *, \dagger$ André Grand ${ }^{a}$ and Bernd Pilawa ${ }^{b}$
}

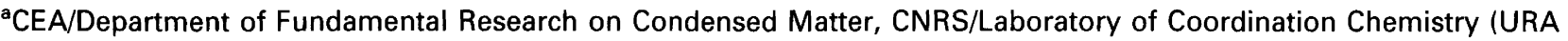
1194), SCIB, Grenoble Centre for Nuclear Studies, F-38054 Grenoble, France and ' $P$ Physics Institute, University of Karlsruhe (TH), D-76 128 Karlsruhe 1, Germany

\begin{abstract}
Öhrström, L., Grand, A. and Pilawa, B., 1996. Spin Density Calculations on the Tetraphenylverdazyl Radical and Two Nitroxide Radicals: First and Second Order Spin Polarizations. - Acta Chem. Scand. 50: 458-461. (c) Acta Chemica Scandinavica 1996.
\end{abstract}

One challenging problem in modern synthetic chemistry is the preparation and design of materials with predefined optical, conducting or magnetic properties. ${ }^{1}$ In one subarea, the field of molecular based magnetic materials, organic $\pi$-radicals take a prominent place. ${ }^{2,3}$ The spin density distribution in such molecules may play an important part in determining the magnetic properties. ${ }^{4,5}$ In verdazyl-type radicals the simplest approach considers the unpaired electron to be delocalized in p-orbitals on the four nitrogen atoms. However, experiments show that substantial contributions, of both positive and negative spin density, may be found relatively far away from these atoms. Indeed, the intermolecular antiferromagnetic coupling between 1,3,5,6-tetraphenylverdazyl radicals, 1, has been interpreted as an interaction between spin density on carbon and nitrogen atoms on neighbouring molecules. ${ }^{6}$

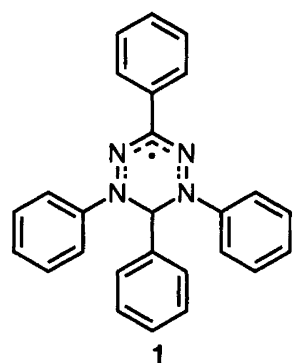

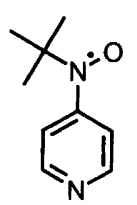

2, NO-4Py

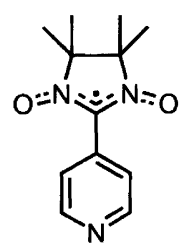

3, NIT-4Py
* To whom correspondence should be addressed.

$\dagger$ Present address: Department of Inorganic Chemistry, Chalmers University of Technology, S-412 96, Göteborg, Sweden.
To understand experimental data correctly it may therefore be essential to know how the spin density is partitioned among the atoms in the molecule. The most important methods in use for the determination of spin distributions are NMR, ESR and polarized neutron diffraction (PND).*

Since all these methods suffer from certain restrictions such as magnetic nuclei in the molecule, time or expensive instrumentation, quantum chemical calculations may become an important complementary tool. Furthermore, we may get insight into the underlying mechanism of the spin distribution.

However, the correct description of the spin density requires the concept of spin polarization. This is an electron correlation effect, and consequently we need more advanced methods than, for example, restricted open-shell HF (doubly occupied MOs and one singly occupied MO for the unpaired electron). In this situation density functional theory, DFT, is an attractive alternative to classical HF methods, since it incorporates most electron correlation effects and it can be used for large molecules. Recent results indicate that DFT methods

\footnotetext{
* It is important to note that these methods do not measure the same aspect of the spin density. Briefly, from ESR we can use the hyperfine coupling constant to extract the spin density that reaches all the way to the nucleus, the Fermi contact density. Moreover, the hyperfine coupling constant to $\mathrm{C}-\mathrm{H}$ hydrogens in $\pi$ systems gives a measure of the $\pi$ spin density on the carbon. The paramagnetic shifts in ${ }^{1} \mathrm{H}-\mathrm{NMR}$ is then a very sensitive probe of the hyperfine interaction. PND on the other hand gives us a total spin density map of the whole molecule. However, some arbitrariness is involved when assigning this spin density to specific atoms.
} 
give spin populations in reasonable agreement with PND experiments. $^{7-9}$

In this study we present DFT calculations on the 1,3,5,6-tetraphenylverdazyl radical. The results are compared to available experimental data and the spin density distribution interpreted by first- and second-order spin polarization. We also include 4-pyridyl-nitroxide (2, NO-4Py) and the 4-pyridylnitronyl-nitroxide (3, NIT-4Py) in our analysis, since they represent another important type of radical, and we can identify the same types of polarization mechanisms as in the verdazyl. In particular the origin, magnitude and sign of the spin density on the pyridyl nitrogen will determine the interaction with a coordinated metal ion. This, in its turn, is important because linking paramagnetic transition metal ions with organic radicals is one approach to molecularbased magnetic materials. ${ }^{10,11}$

\section{Computational details}

The computations were performed using the DGauss $2.3^{12}$ DFT program included in the UniChem package from Cray Research Inc. ${ }^{13}$ At the local spin density level, the functional of Vosko, Wilk and Nusair (VWN) was used for the exchange-correlation potential. ${ }^{14}$ For the non-local corrections to the exchange-correlation energy, the Becke-Perdew (BP) functional, ${ }^{15-17}$ including gradient corrections for exchange ${ }^{16}$ and correlation, ${ }^{17}$ was used in a perturbative way on the LSD SCF density.

The DGauss program uses Gaussian basis sets optimized for LSD calculations. A double $\zeta$ split-valence plus polarization basis set, DZVP, ${ }^{18}$ was used. The contracted basis sets had the following composition: $\mathrm{H}[2 \mathrm{~s}], \mathrm{C}, \mathrm{N}$, $\mathrm{O}[3 \mathrm{~s}, 2 \mathrm{p}, 1 \mathrm{~d}]$. This particular combination of DFT functional and basis sets was recently used with good results. $^{7,9}$

The geometry for the verdazyl 1 was taken from the $\mathrm{X}$-ray diffraction structure, including estimated hydrogen positions. For the 4-pyridyl-nitroxide 2 and the 4-pyridylnitronyl-nitroxide 3 standard bond lengths and angles were used and the molecules were kept planar. All methyl groups were changed to hydrogens.

\section{Results}

The DFT spin populations and the corresponding values from an earlier H-NMR study ${ }^{19}$ are reported in Table 1. A graphical representation of the DFT densities is displayed in Fig. 1, and the two highest occupied molecular orbitals, the SOMO and the $2 \mathrm{HOMO}$, are represented in Fig. 2.*

For atoms without directly bound hydrogens it is not possible to extract spin densities from H-NMR. Instead we have the possibility to compare our calculations to

\footnotetext{
* Although in an unrestricted scheme all orbitals are singly occupied spin orbitals, we retain the term SOMO for the MO containing the unpaired electron.
}

Table 1. Mulliken spin populations of the verdazyl $1 \mathrm{com}$ pared to spin densities from ${ }^{1} \mathrm{H}-\mathrm{NMR}{ }^{a}$

\begin{tabular}{lrr}
\hline Atom & Calc. & \multicolumn{1}{c}{ Exp $^{2}$} \\
\hline C1 & -0.027 & \\
N2 & 0.245 & \\
N3 & 0.164 & \\
C4 & -0.006 & \\
C5 & -0.002 & 0.001 \\
C6 & 0.002 & -0.002 \\
C7 & 0.000 & 0.001 \\
C8 & -0.001 & \\
C9 & -0.014 & 0.053 \\
C10 & 0.048 & -0.019 \\
C11 & -0.011 & 0.050 \\
C12 & 0.055 & \\
C13 & 0.001 & -0.018 \\
C14 & -0.005 & 0.006 \\
C15 & 0.005 & -0.012 \\
C16 & -0.005 & \\
\hline
\end{tabular}

${ }^{a}$ From Ref. 19.

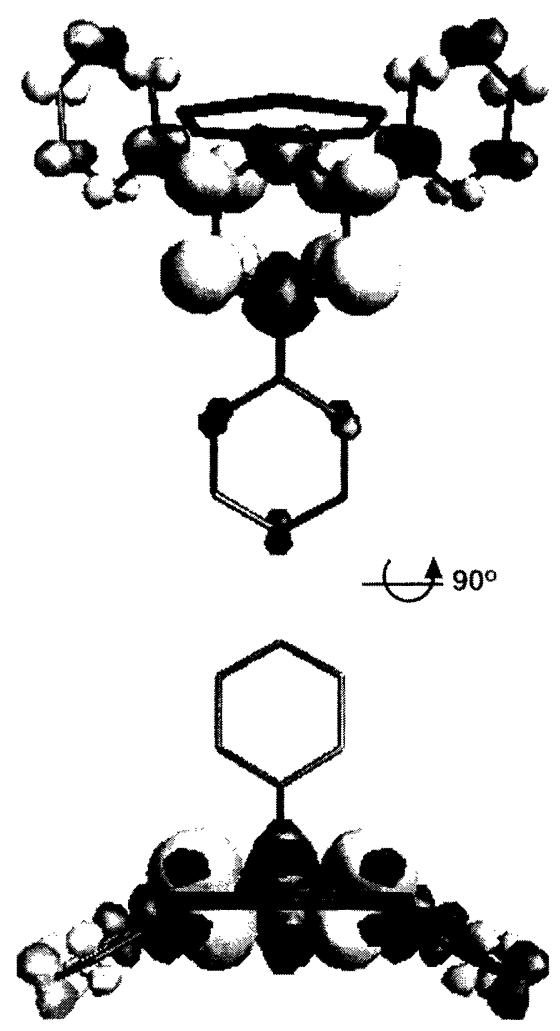

Fig. 1. Spin density for the tetraphenylverdazyl radical 1 calculated by density functional theory. Cut of levels: +0.020 e $\AA^{-3}$ (white) and -0.003 e $\AA^{-3}$ (grey).

EPR measurements. In this case no couplings to the ${ }^{13} \mathrm{C}$ isotope of carbons $1,4,5,9$ or 13 have been reported, but the hyperfine coupling constants to the two different nitrogen atoms were found to be the same. ${ }^{6}$

Unfortunately, the calculation of these isotropic coupling constants is a rather complicated matter, since it requires a very good description not only of the valence shell but also of the core electrons, and it seems that 

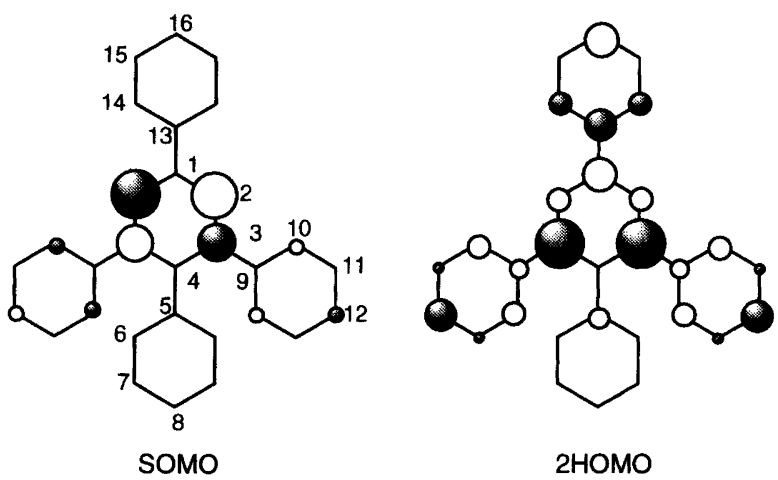

Fig. 2. Representations of the highest singly occupied molecular orbital (SOMO) and the second highest occupied orbital (2HOMO) for the tetraphenylverdazyl radical 1 calculated by density functional theory. The radii of the circles are approximately scaled with the $p_{\mathrm{z}}$-atomic orbital coefficients.

even higher levels of theory are needed to reproduce correctly the experimental values. ${ }^{7,20,21}$ We can nevertheless note that the nitrogen s-spin populations are very close, an indication that the corresponding coupling constants are the same.

Spin populations for NO-4Py and NIT-4Py are reported in Table 2, and the nature of the two highest occupied $\pi$-orbitals are sketched in Fig. 3 .

Table 2. Mulliken spin populations for NO-4Py (2) and NIT-4Py (3).

\begin{tabular}{lrr}
\hline Atom & NO-4Py & NIT-4Py \\
\hline O8 & 0.458 & 0.308 \\
N6 & 0.352 & 0.218 \\
C7 & -0.019 & -0.010 \\
C5 & - & -0.061 \\
C1 & -0.023 & 0.004 \\
C2 & 0.083 & -0.008 \\
C3 & -0.017 & 0.002 \\
N4 & 0.075 & -0.006 \\
C3' & -0.015 & \\
C2 $^{\prime}$ & 0.074 & \\
\hline
\end{tabular}
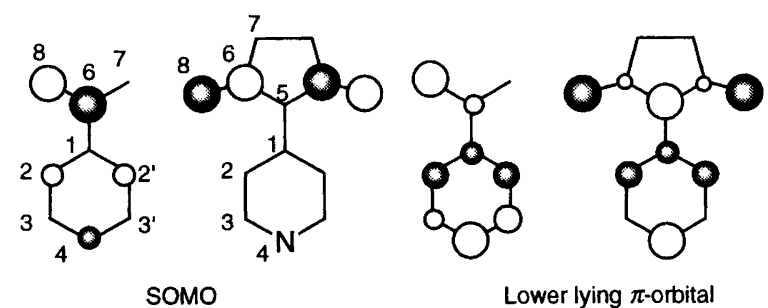

Fig. 3. Representations of the highest singly occupied molecular orbital (SOMO) and the lower $\pi$-orbital that will be spin polarized for the nitroxide radicals NIT-4Py (3) and NO-4Py (2) calculated by density functional theory. The radii of the circles are approximately scaled with the $p_{\mathrm{z}}$-atomic orbital coefficients.

\section{Discussion}

Verdazyl. Since it seems clear that our DFT calculations give a good description of the verdazyl spin density we will only dwell on the mechanisms of spin polarization. The scope and limitations of DFT calculations of spin densities have been discussed elsewhere. ${ }^{22,23}$

In a first approximation we would put all spin density in the highest singly occupied orbital, Fig. 2. Indeed, this approach will account for $98.7 \%$ of the positive spin density in the molecule. However, as much as 0.12 parts of an electron is polarized to have negative spin.

Since the spin populations are almost exclusively of $\pi$-character we will use the allyl radical as model. In this molecule the spin density is not only confined to the two end carbons, negative spin density is also present at the central atom. This is an effect of polarization of the underlying doubly occupied orbital in order to minimize the electron-electron repulsion, a pure correlation effect. $^{24,25}$ (In the extreme case of $100 \%$ polarization of the $\beta$-electron to the central carbon we get three independent spin-MOs with one electron on each carbon, and thus no $\pi$-bonding. What we observe in reality is thus a balance between bonding and electron-electron repulsion.)

What we thus may expect is induced negative spin density on the SOMO's nodal carbon atoms if there is an underlying $\pi$-orbital to polarize. (Note that this $\pi$-orbital also has to coincide to a large extent with the magnetic orbital.) For the carbon atoms $\mathrm{C} 9$ and $\mathrm{C} 11$ this works fine as well as for carbons C1, C5 and C14. These negative spin densities can all be explained by polarization of the 2HOMO (Fig. 2). However, this orbital also has nodes, and a second-order polarization will take place of yet another, lower, MO. This effect will be much smaller, and indeed we see that the experimentally determined spin populations have small positive values for $\mathrm{C} 15$, whereas the neighbouring spins on $\mathrm{C} 14$ and $\mathrm{C} 16$ are larger. This difference in magnitude does not show up in the calculations, possibly because we are at the limit of accuracy.

Finally, according to the arguments above $\mathrm{C} 13$ should have a negative spin population in the order of -0.01 . This is not the case, which only shows that the analysis using only the two highest occupied orbitals has its shortcomings. One factor that may be important is the

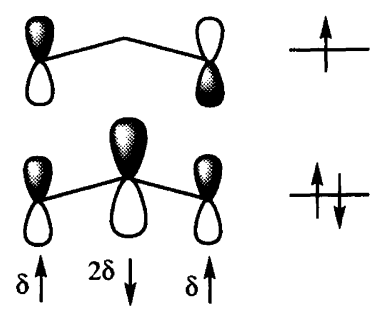

Fig. 4. The highest singly occupied molecular orbital (SOMO) and the second highest occupied orbital (2HOMO) for the allyl radical. The spin polarization of the $2 \mathrm{HOMO}$ is shown. 
polarization of the $\sigma$-bonds, shown by the significant spin density on the $\mathrm{sp}^{3}$-hybridized $\mathrm{C} 4$ carbon, which we have ignored in this interpretation.

For the nitroxides, a planar geometry was used in order to compare the two molecules under the same circumstances. This is also the condition where we expect the largest spin transfers to the pyridyl nitrogen and thus this case will represent an upper limit for the magnetic interaction of the radical with a coordinated metal.

NO-4Py (2). First we note the familiar pattern of alternating signs on the aromatic ring. Furthermore, as is evident from the molecular orbitals of NO-4Py (Fig. 3) the spin density on the pyridyl nitrogen comes from the direct participation of nitrogen p-orbitals in the SOMO. Consequently this is a normal delocalization, not a true polarization effect.

$N I T-4 P y$ (3). In contrast, the pyridyl nitrogen in the nitronylnitroxide 3 does not participate in the magnetic orbital. Even more striking is that there is no significant delocalization on the pyridyl ring. We conclude that the negative spin density on the ring comes from a firstorder spin polarization and that the smaller populations arise from a second-order effect. As in the case of the verdazyl this is in agreement with the shape of the lower $\pi$-orbital for all spin populations in the ring except for the ipso-carbon.

NO-4Py and NIT-4Py bound to Mn(II). When it comes to the coupling of the pyridyl-nitroxides with transitionmetal ions, the large difference in magnitude and sign of NO-4Py and NIT-4Py coupled to Mn(II) has already been noted by Iwamura and co-workers. ${ }^{26,27}$ The reason for this should now be clearer; in the case of $\mathrm{Mn}(\mathrm{hfac})_{2}(\mathrm{NO}-4 \mathrm{Py})_{2}$ we have direct delocalization of the unpaired nitroxide electron and an antiferromagnetic coupling of $8.6 \mathrm{~cm}^{-1}$. In $\mathrm{Mn}(\mathrm{hfac})_{2}$ (NIT-4Py $)_{2}$ a firstorder spin polarization is responsible for the spin density on the coordinated nitrogen. This effect is smaller (note though that we have no way to say this a priori), so the interaction is weaker and, what is more important, the spin polarization reverses the sign so that we now have a ferromagnetic interaction of $0.5 \mathrm{~cm}^{-1}$. We can also note that the ratio of the absolute couplings, 17 , corresponds well to the ratio of the two spin densities, 12, given the experimental error and the approximate geometries used.

Acknowledgements. We thank the Commissariat à l'Energie Atomique for the use of the CRAY-C94 supercomputer in Grenoble. L.Ö. gratefully acknowledges support from the Swedish Natural Science Research Council (NFR).

\section{References}

1. Miller, J. S. and Epstein, A. J. Angew. Chem., Int. Ed. Engl. 33 (1994) 385 and references therein.

2. Kinoshita, M. Jpn. J. Appl. Phys. 33 (1994) 5718.

3. Veciana, J. Organic Magnetic Materials; In: Localized and Itinerant Molecular Magnetism, Coronado, E., Delhaes, P., Gatteschi, D. and Miller, J. S. Eds., NATO ASI, 1995. In press.

4. Kollmar, C. and Kahn, O. Acc. Chem. Res. 26 (1993) 259-265.

5. Kahn, O. Comments Cond. Mat. Phys. 17 (1994) 39.

6. Dormann, E., Dyakonow, W., Gotschy, B., Lang, A., Naarmann, H., Pilawa, B., Walker, N. and Winter, H. Synth. Meth. 55-57 (1993) 3273.

7. Zheludev, A., Barone, V., Bonnet, M., Delley, B., Grand, A., Ressouche, E., Rey, P., Subra, R. and Schweizer, J. J. Am. Chem. Soc. 116 (1994) 2019.

8. Zheludev, A., Grand, A., Ressouche, E., Schweizer, J., Morin, B. G., Epstein, A. J., Dixon, D. A. and Miller, J. S. J. Am. Chem. Soc. 116 (1994) 7243.

9. Zheludev, A., Bonnet, M., Delley, B., Grand, A., Luneau, D., Öhrström, L., Ressouche, E., Rey, P. and Schweizer, J. J. Magn. Magn. Mater. 145 (1995) 293.

10. Caneschi, A., Gatteschi, D., Sessoli, R. and Rey, P. Acc. Chem. Res. 22 (1989) 392-398.

11. Luneau, D., Risoan, G., Rey, P., Grand, A., Caneschi, A., Gatteschi, D. and Laugier, J. Inorg. Chem. 32 (1993) 5616.

12. Andzelm, J. and Wimmer, E. J. Chem. Phys. 96 (1992) 1280.

13. UniChem 2.3; Cray Research, Inc. 2360 Pilot Knob Road, Mendota Heights, MN 551201994.

14. Vosko, S. H., Wilk, L. and Nusair, M. Can. J. Phys. 58 (1980) 1200.

15. Becke, A. D. J. Chem. Phys. 88 (1988) 2547.

16. Becke, A. D. Phys. Rev. A 38 (1988) 3098

17. Perdew, J. P. Phys. Rev. B 33 (1986) 8822.

18. Godbout, N., Salahub, D. R., Andzelm, J. and Wimmer, E. Can. J. Chem. 70 (1992) 560.

19. Lang, A., Naarmann, H., Walker, N. and Dormann, E. Synth. Met. 53 (1993) 379.

20. Barone, V., Grand, A., Luneau, D., Rey, P., Minichino, C. and Subra, R. Nouv. J. Chim. 17 (1993) 545.

21. Barone, V., Adamo, C., Grand, A., Brunel, Y., Fontecave, M. and Subra, R. J. Am. Chem. Soc. 117 (1995) 1083.

22. Yamanaka, S., Kawakami, T., Nagao, H. and Yamaguchi, K. Mol. Cryst. Liq. Cryst. 271 (1995) 19.

23. Yamanaka, S., Kawakami, T., Yamada, S., Nagao, H., Nakano, M. and Yamaguchi, K. Chem. Phys. Lett. 240 (1995) 268.

24. Borden, W. T. Modern Molecular Orbital Theory for Organic Chemists. Prentice-Hall, Englewood Cliffs, NJ 1975.

25. For a detailed treatment of the allyl radical see: Glaser, $\mathbf{R}$. and Choy, G. S.-C. J. Phys. Chem. 98 (1995) 11379.

26. Caneschi, A., Ferraro, F., Gatteschi, D., Rey, P. and Sessoli, R. Inorg. Chem. 29 (1990) 4217.

27. Kitano, M., Ishimaru, Y., Inoue, K., Koga, N. and Iwamura, H. Inorg. Chem. 33 (1994) 6012.

Received 24 July, 1995. 